\title{
Spectrum and vibrational predissociation of the HF dimer. II. Photodissociation cross sections and product state distributions
}

\author{
G. W. M. Vissers, G. C. Groenenboom, and A. van der Avoird ${ }^{\text {a) }}$ \\ Institute of Theoretical Chemistry, NSRIM Center, University of Nijmegen, Toernooiveld, 6525 ED Nijmegen, \\ The Netherlands
}

(Received 30 December 2002; accepted 2 April 2003)

\begin{abstract}
We study vibrational predissociation of the HF dimer both by a full coupled channels treatment as well as in the Fermi golden rule approximation. Photodissociation cross sections, linewidths, and rotational state distributions are computed for excitations from the ground state with rotational quantum numbers $J=1, K=0$ to monomer stretch excited states with $J=K=0$, both for even and odd permutation symmetry. The resonances investigated include excitation of the hydrogen bond donor and acceptor stretches, as well as combinations of one of these modes with the dimer stretch and dimer geared-bending modes. We find that dissociation is sufficiently slow for the Fermi golden rule approximation to be valid. The resonance positions and line strengths are compared with quasibound state calculations. The agreement with experimental data is fairly good for the photofragment angular distributions that were determined from the rotational state distributions, less good for some of the linewidths. Since we carefully checked that the results are converged with respect to the number of vibrational and rotational channels included, the remaining discrepancies are almost certainly due to small deficiencies in the SO-3 potential used in the calculations. (C) 2003 American Institute of Physics. [DOI: 10.1063/1.1577112]
\end{abstract}

\section{INTRODUCTION}

Excitation of the vibrational mode of one of the monomers in $(\mathrm{HF})_{2}$ results in states that lie well above the dissociation limit of the dimer. Since these states are rather longlived, one can approximate them as bound states. This was the approach taken in the preceding paper $^{1}$ (hereafter called Paper I), and for $(\mathrm{HF})_{2}$ this results in a set of vibrational frequencies that are in good agreement with experimental data. However, for a better description of the system one should treat the dissociation process.

Much experimental effort has gone into the study of vibrational predissociation of the HF dimer, resulting in measurements of the predissociation linewidth, ${ }^{2-13}$ and photofragment angular distributions. ${ }^{14-16}$ The first computation of the vibrational predissociation of the HF dimer was done by Halberstadt et al., ${ }^{17}$ who performed a three-dimensional calculation in the Fermi golden rule (FGR) approximation, in which one of the molecules was treated as an atom and the monomer bond length of the other molecule was kept fixed. Later calculations by Zhang et al. ${ }^{18-20}$ extended this to fourdimensional FGR calculations in which both monomer bond lengths were kept fixed. The only full-dimensional (sixdimensional) calculations to date have been reported by Zhang, $\mathrm{Wu}$, and Zhang, ${ }^{21}$ who calculated vibrational predissociation lifetimes for the HF-DF complex using a timedependent golden rule approach.

In this paper we present the results of full-dimensional coupled channels calculations on the vibrational predissociation of $(\mathrm{HF})_{2}$ for excitations from the $J=1, K=0$ ground state to monomer stretch excited states with $J=K=0$. We

${ }^{a)}$ Electronic mail: avda@theochem.kun.nl calculated photodissociation cross sections and rotational state distributions upon excitation of the donor or the acceptor stretch, and combinations of these with excitations in the dimer stretch or dimer geared bend mode. From the cross sections we obtained lifetimes for the resonances investigated. From the calculated rotational state distributions we determined the photofragment angular distributions. ${ }^{15,16,22}$ No calculations were done on dissociation into scattering states with higher $J$, since experimentally it is shown that for given $K$ the lifetimes and product state distributions are independent of $J .^{12,13,16}$

Since dissociation is relatively slow for the HF dimer, we also calculated lifetimes and rotational state distributions using a FGR expression. All calculations were done for both even and odd permutation symmetry.

\section{THEORY}

The dimer stretch and angular basis functions that were used are described in Paper I. The monomer stretch basis functions in Paper I are eigenfunctions of a rotationindependent reference Hamiltonian, and the total basis is a direct product of the angular basis, the dimer stretch and the monomer stretch bases. In photodissociation calculations, matching the total wave function against plane waves requires a basis of fragment eigenfunctions. Hence, in order to obtain the correct boundary conditions for this system, we now choose monomer stretch functions $\chi_{v_{X} j_{X}}\left(r_{X}\right), X=A, B$ that are eigenfunctions of the total monomer Hamiltonian

$$
\hat{h}_{X}=-\frac{\hbar^{2}}{2 \mu_{X}} \frac{1}{r_{X}^{2}} \frac{\partial^{2}}{\partial r_{X}^{2}} r_{X}+\frac{\hat{j}_{X}^{2}}{2 \mu_{X} r_{X}}+V_{X}\left(r_{X}\right)
$$


where $\mu_{X}$ is the reduced mass of the monomer, and $V_{X}$ is the monomer potential. The associated eigenvalues are denoted as $\epsilon_{v_{X} j_{X}}$. Note that these functions are no longer solely dependent on the vibrational quantum number $v_{X}$, but also on the rotational quantum number $j_{X}$.

In the same way as in Paper I, the Hamiltonian is split into two parts, $\hat{H}=\hat{H}_{0}+V_{I}\left(R, \mathbf{r}_{A}, \mathbf{r}_{B}\right)$, where $V_{I}$ is the interaction potential and where $\hat{H}_{0}$ contains the monomer Hamiltonians and the dimer kinetic energy operator:

$$
\hat{H}_{0}=\hat{h}_{A}+\hat{h}_{B}-\frac{\hbar^{2}}{2 \mu} \frac{1}{R} \frac{\partial^{2}}{\partial R^{2}} R+\frac{\hat{J}^{2}+\hat{j}_{A B}^{2}-2 \hat{\mathbf{j}}_{A B} \cdot \mathbf{J}}{2 \mu R^{2}} .
$$

The $R$ dependence of the wave function is represented on a grid in the photodissociation calculations instead of being expanded in a dimer stretch basis. Thanks to the use of monomer eigenfunctions in the monomer stretch basis, the expression for the $R$-dependent matrix elements of $\hat{H}_{0}$ becomes simpler:

$$
\begin{aligned}
\left\langle v_{A}^{\prime} v_{B}^{\prime}\right. & \left.\left(j_{A}^{\prime} j_{B}^{\prime}\right) j_{A B}^{\prime} K^{\prime} ; J M\left|\hat{H}_{0}\right| v_{A} v_{B}\left(j_{A} j_{B}\right) j_{A B} K ; J M\right\rangle \\
= & \delta_{v_{A}^{\prime} v_{A}} \delta_{v_{B}^{\prime} v_{B}} \delta_{j_{A}^{\prime} j_{A}} \delta_{j_{B}^{\prime} j_{B}} \delta_{j_{A B}^{\prime} j_{A B}} \\
& \times\left\{\delta _ { K ^ { \prime } K } \left[\epsilon_{v_{A} j_{A}}+\epsilon_{v_{B} j_{B}}+\frac{\hbar^{2}}{2 \mu R^{2}}[J(J+1)\right.\right. \\
& \left.\left.+j_{A B}\left(j_{A B}+1\right)-2 K^{2}\right]\right]-\frac{\hbar^{2}}{2 \mu R^{2}}\left[\delta_{K^{\prime}, K+1} C_{j_{A B}{ }_{K}}^{+} C_{J K}^{+}\right. \\
& \left.\left.+\delta_{K^{\prime}, K-1} C_{j_{A B} K}^{-} C_{J K}^{-}\right]\right\},
\end{aligned}
$$

where the Coriolis coupling terms $C_{l K}^{ \pm}$are defined in Paper I. Since the effect of these terms is very small for low values of $J$, they are ignored in the calculations, so that $\hat{H}_{0}$ is diagonal in $K$. The matrix elements of the potential in the body fixed basis are the same as in Paper I, except that the radial part no longer contains an integral over $R$, and has become dependent on the monomer rotational quantum numbers. Hence the factor $\left\langle n^{\prime} v_{A}^{\prime} v_{B}^{\prime}\left|c_{L_{A} L_{B} L}\right| n v_{A} v_{B}\right\rangle$ should be replaced by $\left\langle v_{A}^{\prime} j_{A}^{\prime} v_{B}^{\prime} j_{B}^{\prime}\left|c_{L_{A} L_{B} L}\right| v_{A} j_{A} v_{B} j_{B}\right\rangle$.

The partial integral photodissociation cross section for a transition from an initial (bound) state $|i\rangle$ to a scattering state with monomer rotational quantum numbers $\left(j_{A}, j_{B}\right)$ in the coupled channels (CC) calculations can be written as ${ }^{23}$

$$
\sigma_{j_{A} j_{B}}^{(J)}(\omega)=\sum_{j_{A B} l M} \frac{\pi \omega}{c \epsilon_{0}}\left|\left\langle i|\mathbf{e} \cdot \hat{\mu}| \psi_{J M}^{(-) j_{A} j_{B} j_{A B} l}\right\rangle\right|^{2},
$$

where $\mathbf{e}$ is a unit vector in the direction of the electric field of the laser beam, and $\hat{\boldsymbol{\mu}}$ is the transition dipole moment operator of the system. Since the resonances investigated are very narrow, the dissociating states have a well-defined $J$ quantum number. In this paper we only look at excitations from the $J=1, K=0$ ground state to $J=0$ dissociating states. Therefore, the $J$ label on the cross section will henceforth be omitted. To be able to write the energy normalized scattering wave function $\psi_{J M}^{(-) j_{A} j_{B} j_{A B} l}$ in terms of analytically known functions, it is expanded here in a space fixed basis $\left|v_{A} v_{B}\left(j_{A} j_{B}\right) j_{A B} l ; J M\right\rangle^{\mathrm{SF}}$, where we have introduced the end- over-end angular momentum quantum number $l$. This space fixed basis is related to the body fixed basis in which the calculations were performed, via a unitary transformation:

$$
\begin{aligned}
\left|v_{A} v_{B}\left(j_{A} j_{B}\right) j_{A B} l ; J M\right\rangle^{\mathrm{SF}}= & \sum_{K}\left|v_{A} v_{B}\left(j_{A} j_{B}\right) j_{A B} K ; J M\right\rangle \\
& \times \sqrt{\frac{2 l+1}{2 J+1}}\left\langle J K \mid j_{A B} K ; l 0\right\rangle .
\end{aligned}
$$

The expansion of the scattering wave functions can then be written as

$$
\begin{aligned}
& \psi_{J M}^{(-) j_{A} j_{B} j_{A B} l}\left(\mathbf{R}, \mathbf{r}_{A}, \mathbf{r}_{B}\right)=\sum_{\substack{v_{A}^{\prime} v_{B}^{\prime} j_{A}^{\prime} j_{B}^{\prime} \\
j_{A B}^{\prime} l^{\prime}}} R^{-1} f_{v_{A}^{\prime} v_{B}^{\prime} j_{A}^{\prime} j_{B}^{\prime} j_{A B}^{\prime} l^{\prime}}^{j_{B} j_{A B} l}(R) \\
& \times\left|v_{A}^{\prime} v_{B}^{\prime}\left(j_{A}^{\prime} j_{B}^{\prime}\right) j_{A B}^{\prime} l^{\prime} ; J M\right\rangle^{\mathrm{SF}},
\end{aligned}
$$

where the $J$ label on the expansion coefficients is omitted. The upper indices of the $f_{v_{A}^{\prime} v_{B}^{\prime} j_{B}^{\prime} j_{A}^{\prime} j_{B}^{\prime} j_{A B}^{\prime} l^{\prime}}^{j^{\prime}}$ label the different solutions to the Schrödinger equation for energy $E=\hbar \omega$, whereas the lower indices label the basis functions. The solutions are not labeled with $v_{A}$ and $v_{B}$, because in the dissociating function only channels with $v_{A}=v_{B}=0$ are open. The photodissociation boundary conditions for large $R$ read

$$
\begin{aligned}
f_{v_{A}^{\prime} v_{B}^{\prime} j_{A}^{\prime} j_{B}^{\prime} j_{A B}^{\prime} l^{\prime}}^{j_{A} j_{B} j_{A B} l}(R) & \\
= & \frac{1}{\sqrt{2 \pi \hbar}}\left[v_{v_{A}^{\prime} v_{B}^{\prime} j_{A}^{\prime} j_{B}^{\prime} l^{\prime}}(R) \delta_{v_{A}^{\prime} 0} \delta_{v_{B}^{\prime} 0} \delta_{j_{A}^{\prime} j_{A}} \delta_{j_{B}^{\prime} j_{B}} \delta_{j_{A B}^{\prime} j_{A B}} \delta_{l^{\prime} l}\right. \\
& \left.-u_{v_{A}^{\prime} v_{B}^{\prime} j_{A}^{\prime} j_{B}^{\prime} l^{\prime}}(R) S_{v_{A}^{\prime} v_{B}^{\prime} j_{A}^{\prime} j_{B}^{\prime} j_{A B}^{\prime} l^{\prime}, 00 j_{A} j_{B} j_{A B} l}\right],
\end{aligned}
$$

where $\mathbf{S}$ is the scattering matrix. ${ }^{24}$ The flux normalized outgoing waves $v_{v_{A} v_{B} j_{A} j_{B} l}(R)$ and incoming waves $u_{v_{A} v_{B} j_{A} j_{B} l}(R)$ are given by

$$
\begin{aligned}
v_{v_{A} v_{B} j_{A} j_{B} l}(R) & =i \sqrt{\frac{\mu k_{v_{A} v_{B} j_{A} j_{B}}}{\hbar}} R h_{l}^{(1)}\left(k_{v_{A} v_{B} j_{A} j_{B}} R\right), \\
u_{v_{A} v_{B} j_{A} j_{B} l}(R) & =-i \sqrt{\frac{\mu k_{v_{A} v_{B} j_{A} j_{B}}}{\hbar}} R h_{l}^{(2)}\left(k_{v_{A} v_{B} j_{A} j_{B}} R\right) \\
& =v_{v_{A} v_{B} j_{A} j_{B} l}(R)^{*},
\end{aligned}
$$

where $h_{l}^{(1)}$ and $h_{l}^{(2)}$ are spherical Hankel functions of the first and second kind, ${ }^{25}$ respectively, and where the wave numbers $k_{v_{A} v_{B} j_{A} j_{B}}$ are defined as

$$
k_{v_{A} v_{B} j_{A} j_{B}}=\sqrt{\frac{2 \mu\left(E-\epsilon_{v_{A} j_{A}}-\epsilon_{v_{B} j_{B}}\right)}{\hbar^{2}}} .
$$

Just as the potential, the components of the dipole moment are expanded in terms of angular basis functions 


$$
\begin{aligned}
\hat{\mu}_{m}= & \sum_{L_{A} L_{B} L k} d_{L_{A} L_{B} L k}\left(R, r_{A}, r_{B}\right) D_{m k}^{(1)}(\alpha, \beta, 0) * \\
& \times \sum_{M_{A} M_{B}} C_{M_{A}}^{\left(L_{A}\right)}\left(\theta_{A}, \phi_{A}\right) C_{M_{B}}^{\left(L_{B}\right)}\left(\theta_{B}, \phi_{B}\right) \\
& \times\left\langle L_{A} M_{A} L_{B} M_{B} \mid L k\right\rangle .
\end{aligned}
$$

Using this expansion, the matrix elements of $\hat{\mu}_{m}$ in the body fixed basis become

$$
\begin{aligned}
&\left\langle v_{A}^{\prime} v_{B}^{\prime}\left(j_{A}^{\prime} j_{B}^{\prime}\right) j_{A B}^{\prime} K^{\prime} ; J^{\prime} M^{\prime}\left|\hat{\mu}_{m}\right| v_{A} v_{B}\left(j_{A} j_{B}\right) j_{A B} K ; J M\right\rangle \\
&=\left[j_{A}^{\prime}\right]\left[j_{B}^{\prime}\right]\left[j_{A B}^{\prime}\right]\left[J^{\prime}\right]\left[j_{A}\right]\left[j_{B}\right]\left[j_{A B}\right][J] \\
& \times(-1)^{j_{A}+j_{B}+j_{A B}-M^{\prime}}\left(\begin{array}{ccc}
J^{\prime} & 1 & J \\
-M^{\prime} & m & M
\end{array}\right) \\
& \times \sum_{k}\left(\begin{array}{ccc}
J^{\prime} & 1 & J \\
-K^{\prime} & k & K
\end{array}\right) \sum_{L_{A} L_{B} L}[L](-1)^{-L_{A}-L_{B}+L} \\
& \times\left\langle v_{A}^{\prime} j_{A}^{\prime} v_{B}^{\prime} j_{B}^{\prime}\left|d_{L_{A} L_{B} L k}\right| v_{A} j_{A} v_{B} j_{B}\right\rangle \\
& \times\left(\begin{array}{ccc}
j_{A}^{\prime} & L_{A} & j_{A} \\
0 & 0 & 0
\end{array}\right)\left(\begin{array}{ccc}
j_{B}^{\prime} & L_{B} & j_{B} \\
0 & 0 & 0
\end{array}\right)\left(\begin{array}{ccc}
j_{A B}^{\prime} & L & j_{A B} \\
-K^{\prime} & k & K
\end{array}\right) \\
& \times\left\{\begin{array}{ccc}
j_{A}^{\prime} & L_{A} & j_{A} \\
j_{B}^{\prime} & L_{B} & j_{B} \\
j_{A B}^{\prime} & L & j_{A B}
\end{array}\right\},
\end{aligned}
$$

where $[l] \equiv \sqrt{2 l+1}$. Since the transition dipole moment of the HF molecule is large, we only include the effect of the transition dipole moments of the monomers in the expansion, so that the coefficients can be approximated by ${ }^{26}$

$$
\begin{aligned}
d_{L_{A} L_{B} L k}\left(R, r_{A}, r_{B}\right)= & \mu_{\mathrm{HF}}\left(r_{A}\right) \delta_{L_{A} 1} \delta_{L_{B} 0} \delta_{L_{A} L} \\
& +\mu_{\mathrm{HF}}\left(r_{B}\right) \delta_{L_{A} 0} \delta_{L_{B} 1} \delta_{L_{B} L} .
\end{aligned}
$$

Assuming the integral over these coefficients to be independent of the monomer rotational quantum number, we get

$$
\begin{aligned}
& \left\langle v_{A}^{\prime} j_{A}^{\prime} v_{B}^{\prime} j_{B}^{\prime}\left|d_{L_{A} L_{B} L k}\right| v_{A} j_{A} v_{B} j_{B}\right\rangle \\
& =\left\langle 1\left|\mu_{\mathrm{HF}}\right| 0\right\rangle \delta_{v_{A}^{\prime}{ }^{0}} \delta_{v_{B}^{\prime} 0}\left[\delta_{v_{A} 1} \delta_{v_{B} 0} \delta_{L_{A} 1} \delta_{L_{B} 0} \delta_{L_{A} L}\right. \\
& \left.\quad+\delta_{v_{A} 0} \delta_{v_{B} 1} \delta_{L_{A} 0} \delta_{L_{B} 1} \delta_{L_{B} L}\right] .
\end{aligned}
$$

The total cross section $\sigma^{\text {tot }}(\omega)$ is obtained by summing the partial cross sections over all $j_{A}$ and $j_{B}$. In the neighborhood of a resonance, $\sigma^{\text {tot }}(\omega)$ takes the form of a Lorentzian

$$
\sigma^{\text {tot }}(\omega)=f \frac{\Gamma / 2}{\left(\omega-\omega_{r}\right)^{2}+(\Gamma / 2)^{2}},
$$

centered around the resonance frequency $\omega_{r}$, where $\Gamma$ is the full width at half maximum (FWHM) of the line, and $f$ is a proportionality constant. The linewidth $\Gamma$ is inversely proportional to the lifetime $\tau$ of the system, $\Gamma=1 / \tau$, so that by calculating the cross section at a number of frequencies around a certain resonance, and fitting these to a Lorentzian function, we obtain the lifetime of the system for this particular resonance. The fit can also be used in calculating the line strength $S$ for the transition, which is given by

$$
S=\sum_{j_{A} j_{B} j_{A B} l} \int\left|\left\langle i|\mathbf{e} \cdot \hat{\boldsymbol{\mu}}| \psi_{J M}^{(-) j_{A} j_{B} j_{A B} l}\right\rangle\right|^{2} d E .
$$

Since $\Gamma$ is very small compared to $\omega_{r}$, we can reduce this to

$$
S=\frac{\hbar c \epsilon_{0}}{\pi \omega_{r}} \int \sigma^{\mathrm{tot}}(\omega) d \omega=\frac{\hbar c \epsilon_{0}}{\omega_{r}} f .
$$

The dissociation process is slow for this system, so that the linewidth can also be calculated directly in a Fermi golden rule (FGR) approximation:

$$
\Gamma=\sum_{j_{A} j_{B}} \Gamma_{j_{A} j_{B}}=\sum_{j_{A} j_{B} j_{A B} l M} \frac{2 \pi}{\hbar}\left|\left\langle\psi_{b}\left|V_{10}\right| \psi_{J M}^{(-) j_{A} j_{B} j_{A B} l}\right\rangle\right|^{2},
$$

where $V_{10}$ is the vibrational coupling potential between excited $\left(v_{A}+v_{B}=1\right)$ and ground state $\left(v_{A}=v_{B}=0\right)$ functions. This coupling potential consists of $\left\langle v_{A}^{\prime} v_{B}^{\prime}\left|V_{I}\right| v_{A} v_{B}\right\rangle$ $=\left\langle 10\left|V_{I}\right| 00\right\rangle$ and $\left\langle 01\left|V_{I}\right| 00\right\rangle$ matrix elements. The quasibound state $\left|\psi_{b}\right\rangle$ in these calculations is an eigenstate of the Hamiltonian in a basis without the ground state monomer stretch functions. The scattering wave function, which is calculated at the energy of $\left|\psi_{b}\right\rangle$, is in this approximation expanded in a basis with ground state stretch functions only.

For the CC calculations, the angular state distribution is obtained by taking the fractions of the partial cross sections with respect to the total cross section, and is calculated at the resonance energy:

$$
P_{j_{A} j_{B}}=\frac{\sigma_{j_{A} j_{B}}\left(\omega_{r}\right)}{\sigma^{\mathrm{tot}}\left(\omega_{r}\right)} .
$$

A similar expression involving $\Gamma_{j_{A} j_{B}}$ and $\Gamma$ was used in the FGR calculations. From these angular state distributions, the theoretical angular distributions were reconstructed, using the program that Bohac et al. ${ }^{16,22}$ used to fit an angular state distribution to their experimental data.

\section{COMPUTATIONAL DETAILS}

Since we found in Paper I that the SO-3 potential energy surface by Klopper et al $^{27}$ is of very high quality when computing (quasi)bound states, this is the potential with which all calculations were performed.

The coupled channels calculations were performed in a basis with $j_{A}^{\max }=j_{B}^{\max }=13$ and $v_{A}+v_{B} \leqslant 2$, leading to a total of 3150 channels, of which between 175 and 232 channels were open in the investigated energy range. In the Fermi golden rule calculations, basis sets with the same vibrational basis and $j_{A}^{\max }=13$ and 16 were used, where the latter basis contained approximately 5500 channels in total. All calculations were done in the helicity decoupled approximation, neglecting Coriolis coupling off-diagonal in $K$.

The scattering wave function was propagated outwards using the renormalized Numerov ${ }^{28}$ propagator, on an equally spaced grid of 263 points in the range $2-18 a_{0}$, which was tested to be sufficient to converge the calculated properties. The integral was built up in parallel to the propagation, using a method similar to that described by Gadéa et al. ${ }^{29}$ In the 
TABLE I. Vibrational predissociation linewidths for $(\mathrm{HF})_{2}$ (in $\mathrm{MHz}$ ). Values are obtained from coupled channels (CC) and Fermi golden rule (FGR) calculations, in a basis with $v_{A}+v_{B} \leqslant 2$ and $j_{A}, j_{B} \leqslant j_{A}^{\max }$, and for even $\left(A_{1}\right)$ and odd $\left(B_{2}\right)$ scattering states with respect to monomer exchange. The experimental linewidths are from Ref. 13 .

\begin{tabular}{ccccc}
\hline \hline & CC & FGR & FGR & \\
& $j_{A}^{\max }=13$ & 13 & 16 & Expt. \\
\hline$\nu_{1}$ & 4.00 & $A_{1}$ & & \\
$\nu_{1}+\nu_{4}$ & 15.73 & 4.31 & 6.35 & 6.4 \\
$\nu_{1}+\nu_{5}$ & 12.83 & 17.03 & 18.15 & 25 \\
$\nu_{2}$ & 42.81 & 50.77 & 43.60 & 330 \\
$\nu_{2}+\nu_{4}$ & 77.79 & 90.23 & 82.25 & \\
$\nu_{2}+\nu_{5}$ & 48.08 & 53.65 & 47.75 & 270 \\
& & & & \\
$\nu_{1}$ & 3.72 & $B_{2}$ & & \\
$\nu_{1}+\nu_{4}$ & 10.55 & 10.61 & 11.02 & 40 \\
$\nu_{1}+\nu_{5}$ & 9.09 & 8.99 & 11.25 & 45 \\
$\nu_{2}$ & 37.41 & 44.54 & 47.85 & 330 \\
$\nu_{2}+\nu_{4}$ & 63.34 & 74.60 & 73.33 & 300 \\
$\nu_{2}+\nu_{5}$ & 36.78 & 41.26 & 46.52 & 270 \\
\hline \hline
\end{tabular}

propagation we used the body fixed basis; the unitary transformation to the space fixed basis [Eq. (5)] was not performed until the matching.

The FGR calculations would normally be performed at the energy of the quasibound state used. However, we found that this approximation is not a very good one, especially in the calculation of the angular distributions. Since the $\left(v_{A}, v_{B}\right)=(0,0)$ channels are left out of the basis in the calculation of this quasibound state, the computed eigenvalues will generally be too low. In some cases this led to situations where channels of high internal energy were closed in the FGR approximation, simply because the energy at which the calculation was performed was too low. Therefore, the FGR calculations have been made at the quasibound state energy
TABLE II. Peak positions $E_{r}=\hbar \omega_{r}$ of the resonances from quasibound state calculations (QBS), and coupled channels calculations (CC) on the SO-3 potential. All values are in $\mathrm{cm}^{-1}$, relative to the $A_{1}$ ground state of $-1061.73 \mathrm{~cm}^{-1}$.

\begin{tabular}{cccccc}
\hline \hline & \multicolumn{3}{c}{$A_{1}$} & & \multicolumn{2}{c}{$B_{2}$} \\
\cline { 2 - 3 } \cline { 5 - 6 } & QBS & CC & & QBS & CC \\
\hline$\nu_{1}$ & 3929.17 & 3929.22 & & 3929.01 & 3929.03 \\
$\nu_{1}+\nu_{4}$ & 4056.93 & 4056.92 & & 4055.56 & 4055.55 \\
$\nu_{1}+\nu_{5}$ & 4096.22 & 4096.21 & & 4094.15 & 4094.14 \\
$\nu_{2}$ & 3867.09 & 3867.15 & & 3867.26 & 3867.32 \\
$\nu_{2}+\nu_{4}$ & 4000.50 & 4000.51 & & 4001.39 & 4001.40 \\
$\nu_{2}+\nu_{5}$ & 4043.22 & 4043.23 & & 4045.44 & 4045.45 \\
\hline \hline
\end{tabular}

of the predissociating state involved (see Paper I), which is a good approximation of the true resonance energy.

\section{RESULTS AND DISCUSSION}

The results of the calculations of the linewidths are given in Table I. Shown there are the FWHM linewidths obtained by coupled channels calculations in a basis with $j_{A}, j_{B} \leqslant 13$, and golden rule calculations in the same basis, as well as in a basis with $j_{A}, j_{B} \leqslant 16$. The resonances studied involve excitation of the acceptor stretch $\left(\nu_{1}\right)$ or donor stretch $\left(\nu_{2}\right)$, and combinations of one of these modes with an excitation in the dimer stretch $\left(\nu_{4}\right)$ or dimer geared bend $\left(\nu_{5}\right)$ mode. All calculations have been done for scattering states of even $\left(A_{1}\right)$ and odd $\left(B_{2}\right)$ symmetry with respect to exchange, see Paper I for details on the symmetry labeling. We see that the calculated linewidths from FGR and CC calculations agree reasonably well with each other, indicating that the golden rule approximation is valid for the resonances under investigation. Furthermore, we find from the FGR calculations that increasing the rotational basis from $j_{A}^{\max }=13$ to 16 does not lead to a significant change in the

TABLE III. Overview of the most important contributions to the fragment rotational state distributions for scattering states of $A_{1}$ symmetry. Values are percentages, and are taken from FGR calculation with rotational basis up to $j_{A}^{\max }=j_{B}^{\max }=16$. The last two lines show the distribution (in \%) of the excess energy over fragment rotational $\left(E_{\text {rot }}\right)$ and translational energy $\left(E_{\text {trans }}\right)$.

\begin{tabular}{|c|c|c|c|c|c|c|}
\hline$\left(j_{A}, j_{B}\right)$ & $\nu_{1}$ & $\nu_{1}+\nu_{4}$ & $\nu_{1}+\nu_{5}$ & $\nu_{2}$ & $\nu_{2}+\nu_{4}$ & $\nu_{2}+\nu_{5}$ \\
\hline$(7,5)$ & 0.35 & 0.89 & 1.37 & 3.79 & 5.75 & 0.42 \\
\hline$(9,3)$ & 1.87 & 4.14 & 5.42 & 1.15 & 2.58 & 3.16 \\
\hline$(8,5)$ & 5.81 & 10.37 & 8.17 & 3.95 & 4.63 & 4.61 \\
\hline$(9,4)$ & 4.09 & 11.62 & 6.00 & 14.68 & 13.38 & 2.64 \\
\hline$(8,6)$ & 9.91 & 7.17 & 5.71 & 8.38 & 8.60 & 12.88 \\
\hline$(10,2)$ & 1.07 & 0.56 & 3.26 & 7.59 & 9.68 & 0.98 \\
\hline$(9,5)$ & 14.15 & 10.00 & 11.43 & 5.18 & 5.74 & 24.95 \\
\hline$(10,3)$ & 5.09 & 1.28 & 1.30 & 4.23 & 2.23 & 2.61 \\
\hline$(8,7)$ & 7.20 & 5.30 & 3.54 & 2.48 & 2.62 & 3.72 \\
\hline$(10,4)$ & 9.26 & 6.97 & 10.48 & 8.06 & 11.94 & 9.59 \\
\hline$(11,0)$ & 0.35 & 0.29 & 0.13 & 10.11 & 2.52 & 0.26 \\
\hline$(9,6)$ & 8.43 & 3.59 & 11.75 & 9.21 & 4.23 & 6.06 \\
\hline$(11,1)$ & 6.39 & 8.39 & 1.89 & 2.86 & 2.18 & 3.02 \\
\hline$(11,2)$ & 1.87 & 4.84 & 5.96 & 4.50 & 0.51 & 4.99 \\
\hline$(10,5)$ & 10.75 & 3.62 & 5.71 & 0.00 & 3.95 & 4.76 \\
\hline$E_{\text {rot }}$ & 85.67 & 79.23 & 80.29 & 85.13 & 78.88 & 82.13 \\
\hline$E_{\text {trans }}$ & 14.33 & 20.77 & 19.71 & 14.87 & 21.12 & 17.87 \\
\hline
\end{tabular}


TABLE IV. As in Table III, for scattering states of $B_{2}$ symmetry.

\begin{tabular}{crrrrrr}
\hline \hline$\left(j_{A}, j_{B}\right)$ & \multicolumn{1}{c}{$\nu_{1}$} & $\nu_{1}+\nu_{4}$ & $\nu_{1}+\nu_{5}$ & \multicolumn{1}{c}{$\nu_{2}$} & $\nu_{2}+\nu_{4}$ & $\nu_{2}+\nu_{5}$ \\
\hline$(9,3)$ & 0.26 & 0.24 & 0.23 & 3.49 & 5.96 & 0.54 \\
$(9,4)$ & 5.05 & 7.29 & 1.03 & 2.89 & 3.23 & 4.23 \\
$(10,1)$ & 1.31 & 1.64 & 0.28 & 6.70 & 7.94 & 0.62 \\
$(8,6)$ & 5.25 & 6.42 & 1.09 & 1.75 & 3.73 & 1.39 \\
$(10,2)$ & 8.24 & 5.86 & 1.28 & 23.82 & 21.91 & 1.37 \\
$(9,5)$ & 5.78 & 16.57 & 20.30 & 1.92 & 3.40 & 3.29 \\
$(10,3)$ & 10.97 & 10.78 & 7.76 & 4.79 & 11.69 & 10.28 \\
$(10,4)$ & 14.84 & 8.62 & 18.24 & 13.24 & 6.32 & 2.57 \\
$(11,0)$ & 0.20 & 0.09 & 0.36 & 14.93 & 3.19 & 0.25 \\
$(9,6)$ & 15.45 & 14.97 & 17.75 & 8.16 & 6.76 & 7.10 \\
$(11,1)$ & 1.54 & 0.57 & 2.25 & 1.28 & 4.59 & 11.48 \\
$(11,2)$ & 9.70 & 2.87 & 6.42 & 9.09 & 2.83 & 25.40 \\
$(10,5)$ & 8.93 & 4.91 & 3.43 & 0.00 & 5.31 & 5.21 \\
$(11,3)$ & 0.00 & 1.49 & 8.74 & 0.00 & 2.15 & 10.81 \\
$(9,7)$ & 0.00 & 4.50 & 7.20 & 0.00 & 0.00 & 10.55 \\
$E_{\text {rot }}$ & 88.21 & 83.35 & 87.03 & 88.56 & 82.68 & 90.33 \\
$E_{\text {trans }}$ & 11.79 & 16.65 & 12.97 & 11.44 & 17.32 & 9.67 \\
\hline \hline
\end{tabular}

linewidths. The experimental linewidths are reproduced reasonably well for the $\nu_{1}$ scattering states of $A_{1}$ symmetry. The much larger linewidths of the $\nu_{2}$ states are not reproduced quite so well, although also the calculated line widths for $\nu_{2}$ excited states are larger than their $\nu_{1}$ counterparts. The experimental trend of increase in linewidth of the $\nu_{1}$ states when going from even to odd exchange symmetry is not found in the calculations.

The positions of the resonances, obtained from the Lorentzian fit of the CC cross section, are given in Table II. For comparison, the energy levels from the quasibound state (QBS) calculations of Paper I are also given. The correspondence between the two sets of numbers is very good: the positions of resonances in the continuum agree to within $0.06 \mathrm{~cm}^{-1}$ with the bound state levels, indicating that dissociation is indeed slow.

Tables III and IV give an overview of the most important

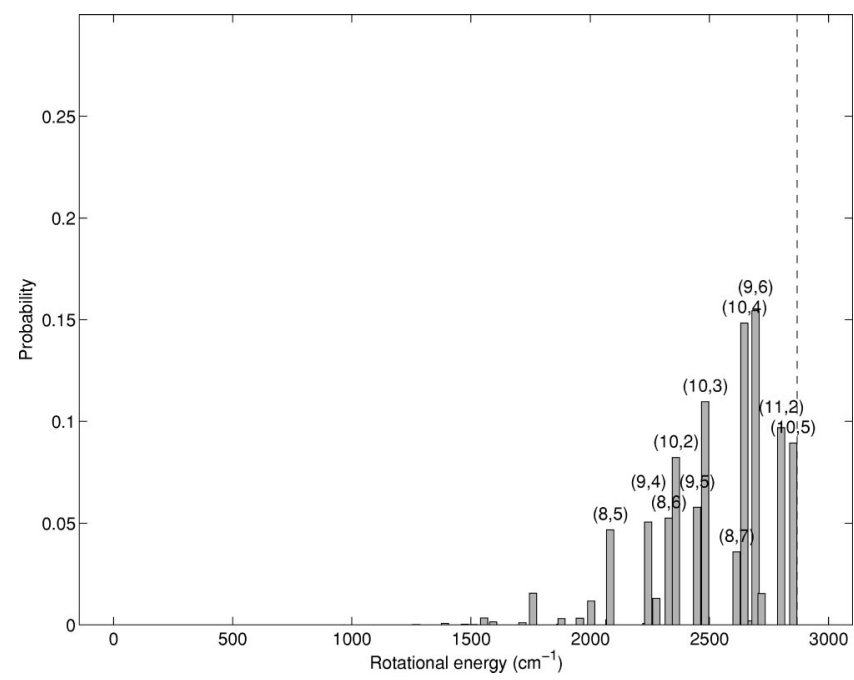

FIG. 1. Rotational state distribution of $(J, K, \Gamma)=\left(1,0, B_{1}\right) \rightarrow\left(0,0, B_{2}\right) \quad \nu_{1}$ transition. Each probability is drawn at the energy of the corresponding channel eigenvalue. The dashed line denotes the total amount of kinetic energy available. contributions to the rotational state distributions for all calculated scattering states. Also the distribution of the excess energy over fragment rotation and translation is given. The values in Tables III and IV are the results of the FGR calculations in the large $\left(j_{A}^{\max }=16\right)$ rotational basis, since we believe these numbers to be the most accurate. The amount of excess energy that goes into fragment rotation ranges from $79 \%$ to $90 \%$. Furthermore, Tables III and IV show that there exist large variations in the rotational state distributions for the different transitions. This is illustrated in Figs. 1 and 2, where the rotational state distribution is plotted for the $\nu_{1}$ and $\nu_{2}$ resonances of odd permutation symmetry. We see that the $\nu_{2}$ distribution is sharply peaked with very strong contributions from the $(10,2),(10,4)$, and $(11,0)$ channels. Other channels dominate in the $\nu_{1}$ resonance, in which the contributions are also distributed more equally.

For some of the calculated scattering states, angular distributions have been reconstructed from the calculated rotational state distributions, to allow for a direct comparison

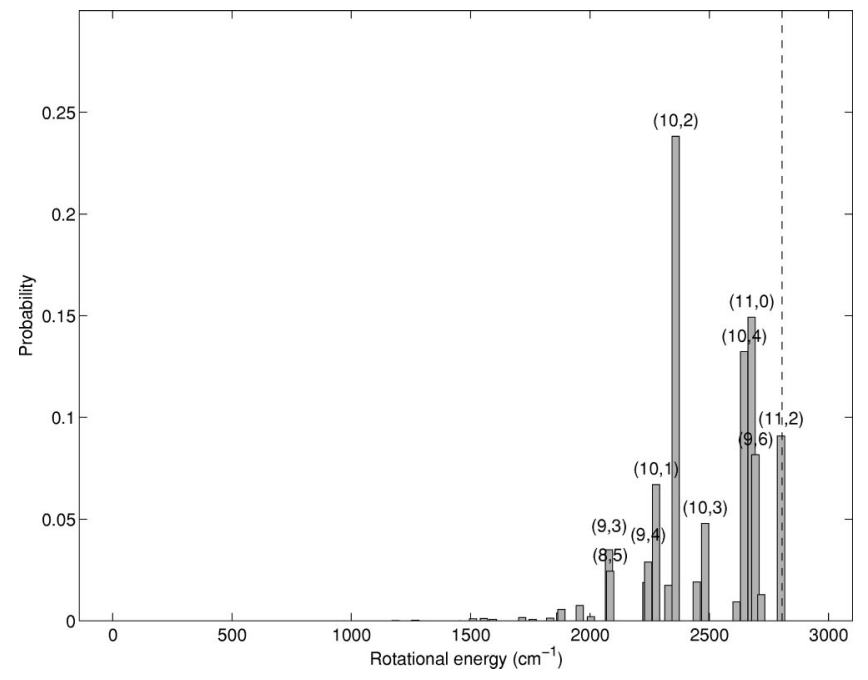

FIG. 2. As in Fig. 1, for $\left(1,0, B_{1}\right) \rightarrow\left(0,0, B_{2}\right) \nu_{2}$ transition. 


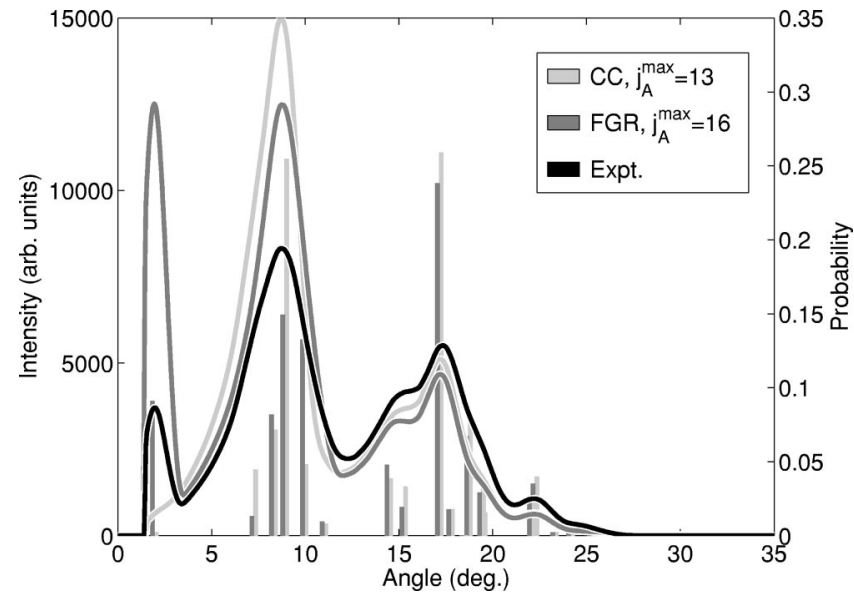

FIG. 3. Angular distribution of the $\left(1,0, B_{1}\right) \rightarrow\left(0,0, B_{2}\right) \quad \nu_{2}$ transition. The experimental data are from Ref. 15.

with the experimental results obtained by Miller and co-workers. ${ }^{15,22}$ These states are the acceptor stretch $\left(\nu_{1}\right)$ and donor stretch $\left(\nu_{2}\right)$ excited states, and combinations of these modes with dimer stretch $\left(\nu_{4}\right)$ and geared bend $\left(\nu_{5}\right)$ excitation, all of $B_{2}$ symmetry. The results are shown in Figs. $3-6$. As can be seen in Figs. 3-6, the positions of the different maxima in the angular distributions are reproduced rather well by the calculations. The effect of the increase in rotational basis from $j_{A}^{\max }=13$ to 16 , which was only done for the FGR calculations, is in most cases not very large. The two main effects of this increase can be seen in Figs. 3 and 6, where it causes two more peaks to appear which are not very noticeable in the $j_{A}^{\max }=13$ results. Although the calculated peak positions agree reasonably well with the experimental data, the intensities sometimes do not, most notably at small angles, where the intensity is very sensitive to the rotational state distribution.

In their paper on the measurement of infrared spectra for bands associated with the $\nu_{4}$ and $\nu_{5}$ vibrations in combination with donor or acceptor stretch, Bohac and Miller ${ }^{22}$ indicated there was a large difference in intensity between the even and odd tunneling components. We have therefore listed in Table V the calculated line strengths, both from

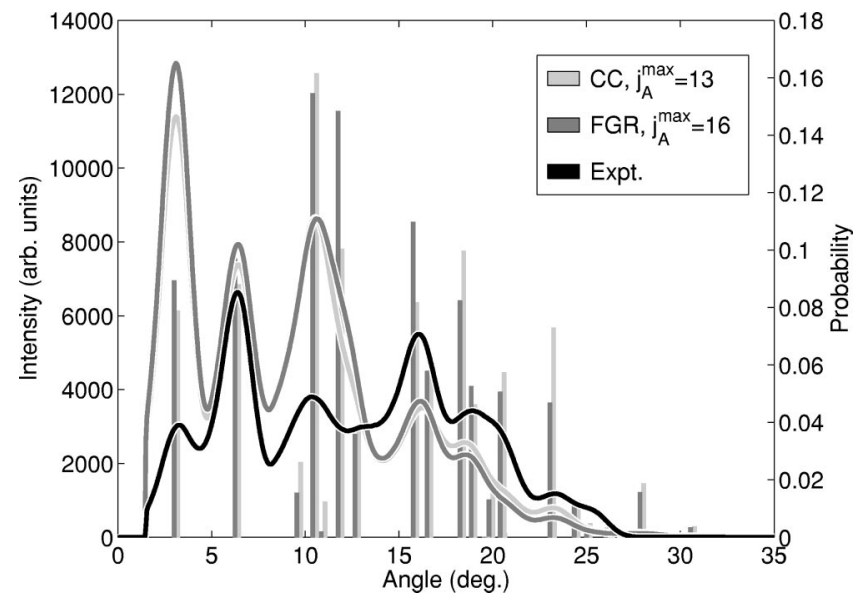

FIG. 4. Angular distribution of the $\left(1,0, B_{1}\right) \rightarrow\left(0,0, B_{2}\right) \nu_{1}$ transition. The experimental data are from Ref. 15.

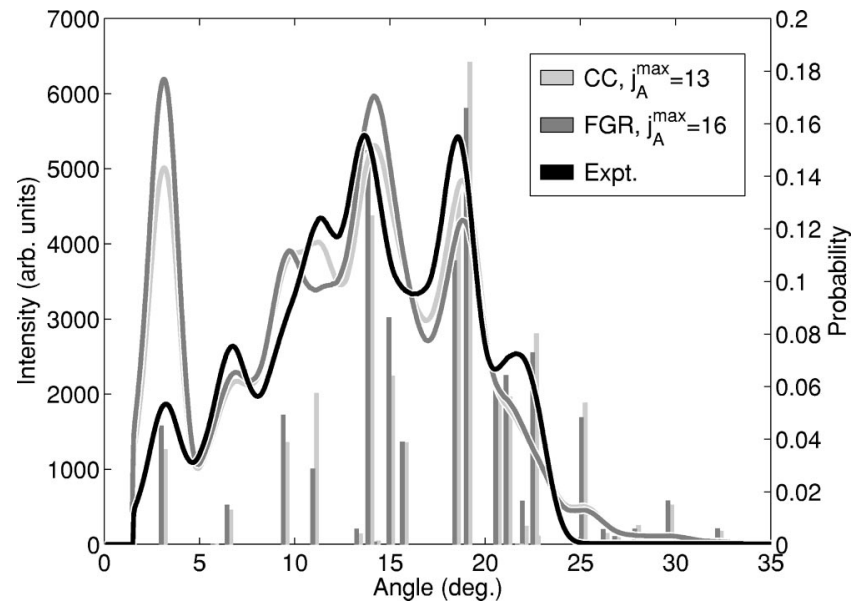

FIG. 5. Angular distribution of the $\left(1,0, B_{1}\right) \rightarrow\left(0,0, B_{2}\right) \nu_{1}+\nu_{4}$ transition. The experimental data are from Ref. 22.

QBS and CC calculations, where the latter were obtained by integrating the Lorentzian line shape of the resonance [see Eq. (17)]. An experimental value ${ }^{30}$ of $0.0388 e a_{0}$ was used for the HF monomer transition dipole moment $\left\langle 1\left|\mu_{\mathrm{HF}}\right| 0\right\rangle$.

The agreement between the results of both calculations is again very good. The relatively large differences between QBS and CC line strengths of the $\nu_{1}$ resonance of $A_{1}$ symmetry and the $\nu_{2}+\nu_{4}$ state of $B_{2}$ symmetry are probably explained by the fact that the contribution of $v_{A}=v_{B}=0$ functions in these calculated quasibound excited states is large (see Paper I). Despite the agreement between CC and QBS calculations, we were unable to reproduce the experimental difference in line strength.

\section{CONCLUSION}

Photodissociation of the HF dimer has been studied in a series of full coupled channels calculation, as well as in a Fermi golden rule approximation. We have calculated linewidths, rotational state distributions, and line strengths for several transitions involving the excitation of the donor or acceptor stretch, and for combinations of these excitations with the dimer stretching and geared bend modes. For four of

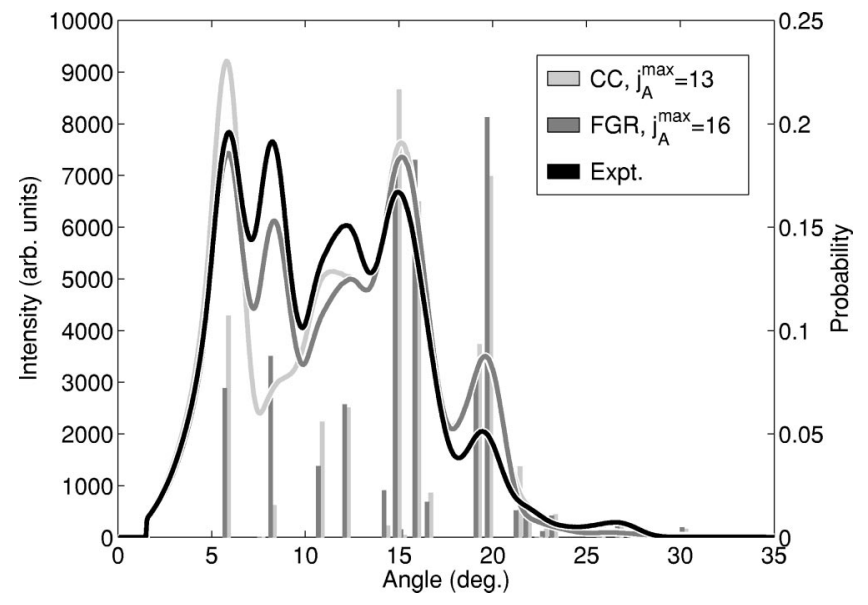

FIG. 6. Angular distribution of the $\left(1,0, B_{1}\right) \rightarrow\left(0,0, B_{2}\right) \nu_{1}+\nu_{5}$ transition. The experimental data are from Ref. 22. 
TABLE V. Line strengths from quasibound state (QBS) and photodissociation calculations (CC), in $10^{-3} e^{2} a_{0}^{2}$.

\begin{tabular}{cccccc}
\hline \hline & \multicolumn{3}{c}{$A_{1}$} & & \multicolumn{2}{c}{$B_{2}$} \\
\cline { 2 - 3 } \cline { 5 - 6 } \cline { 5 - 6 } & QBS & CC & & QBS & CC \\
\hline$\nu_{1}$ & 1.7982 & 1.9442 & & 2.0106 & 2.0104 \\
$\nu_{1}+\nu_{4}$ & 0.0462 & 0.0470 & & 0.1103 & 0.1123 \\
$\nu_{1}+\nu_{5}$ & 0.0330 & 0.0331 & & 0.0451 & 0.0451 \\
$\nu_{2}$ & 3.9986 & 4.0730 & & 4.0016 & 3.9994 \\
$\nu_{2}+\nu_{4}$ & 0.2409 & 0.2416 & & 0.1575 & 0.1775 \\
$\nu_{2}+\nu_{5}$ & 0.1374 & 0.1370 & & 0.0526 & 0.0545 \\
\hline \hline
\end{tabular}

the calculated transitions we have reconstructed the angular distributions from our calculated rotational state distributions, which allows for a direct comparison with the experimental data.

The calculations show that the photodissociation of $(\mathrm{HF})_{2}$ is sufficiently slow for the Fermi golden rule approximation to be valid. This is supported by the fact that the calculated lifetimes and angular distributions from the FGR calculations are not very different from the results of the full coupled channels calculations. Furthermore, the peak positions and line strengths from the scattering calculations agree very well with results of quasibound state calculations, which do not take dissociation into account.

The calculated angular distributions agree fairly well with experimental data, although the relative intensities of the peaks within the distributions are not perfect yet. The experimental linewidths are not reproduced so well, with some linewidths being off by a factor of 8 .

Since the FGR calculations have shown that an increase in the rotational basis does not improve the calculated linewidths and only has a minor effect on the angular distributions, we believe that the remaining problems are most probably due to the SO-3 potential energy surface. The possibility exists that an increase in the monomer stretch basis is required to describe the experimental findings better, but this seems improbable since the $v_{A}+v_{B}=3$ stretch functions are very far away in energy from the resonances investigated.

Although the SO-3 potential is a huge improvement over the older potentials, such as SQSBDE, ${ }^{31}$ it is not good enough to reproduce all experimental data in a photodissociation process. Since many of the features studied in this paper are very sensitive on the exact shape of the potential, the challenge of creating a potential which describes them better remains.

\section{ACKNOWLEDGMENTS}

The authors would like to thank Professor R. E. Miller and Dr. L. Oudejans for the use of their data to calculate the angular distributions, and for their help in doing so. This research has been financially supported by the Council for Chemical Sciences of the Netherlands Organization for Scientific Research (CW-NWO).

${ }^{1}$ G. W. M. Vissers, G. C. Groenenboom, and A. van der Avoird, J. Chem. Phys. 119, 277 (2003), preceeding paper.

${ }^{2}$ J. M. Lisy, A. Tramer, M. F. Vernon, and Y. T. Lee, J. Chem. Phys. 75, 4733 (1981)

${ }^{3}$ A. S. Pine and W. J. Lafferty, J. Chem. Phys. 78, 2154 (1983).

${ }^{4}$ R. L. DeLeon and J. S. Muenter, J. Chem. Phys. 80, 6092 (1984).

${ }^{5}$ A. S. Pine, W. J. Lafferty, and B. J. Howard, J. Chem. Phys. 81, 2939 (1984)

${ }^{6}$ A. S. Pine and B. J. Howard, J. Chem. Phys. 84, 590 (1986).

${ }^{7}$ Z. S. Huang, K. W. Jucks, and R. E. Miller, J. Chem. Phys. 85, 3338 (1986).

${ }^{8}$ A. S. Pine and G. T. Fraser, J. Chem. Phys. 89, 6636 (1988).

${ }^{9}$ K. von Puttkamer and M. Quack, Chem. Phys. 139, 31 (1989).

${ }^{10}$ M. A. Suhm, J. T. Farrell, A. McIlroy, and D. J. Nesbitt, J. Chem. Phys. 97, 5341 (1992).

${ }^{11}$ H.-C. Chang and W. Klemperer, J. Chem. Phys. 98, 9266 (1993).

${ }^{12}$ D. T. Anderson, S. Davis, and D. J. Nesbitt, J. Chem. Phys. 104, 6225 (1996)

${ }^{13}$ D. T. Anderson, S. Davis, and D. J. Nesbitt, J. Chem. Phys. 105, 4488 (1996)

${ }^{14}$ D. C. Dayton, K. W. Jucks, and R. E. Miller, J. Chem. Phys. 90, 2631 (1988).

${ }^{15}$ M. D. Marshall, E. J. Bohac, and R. E. Miller, J. Chem. Phys. 97, 3307 (1992).

${ }^{16}$ E. J. Bohac, M. D. Marshall, and R. E. Miller, J. Chem. Phys. 96, 6681 (1992).

${ }^{17}$ N. Halberstadt, P. Brechignac, J. A. Beswick, and M. Shapiro, J. Chem. Phys. 84, 170 (1986).

${ }^{18}$ D. H. Zhang and J. Z. H. Zhang, J. Chem. Phys. 98, 5978 (1993).

${ }^{19}$ D. H. Zhang and J. Z. H. Zhang, J. Chem. Phys. 99, 6624 (1993).

${ }^{20}$ M. von Dirke, Z. Bačić, D. H. Zhang, Q. Wu, and J. Z. H. Zhang, J. Chem. Phys. 102, 4382 (1995).

${ }^{21}$ D. H. Zhang, Q. Wu, and J. Z. H. Zhang, J. Chem. Phys. 102, 124 (1995).

${ }^{22}$ E. J. Bohac and R. E. Miller, J. Chem. Phys. 99, 1537 (1993).

${ }^{23}$ R. Schinke, Photodissociation Dynamics (Cambridge University Press, Cambridge, 1993).

${ }^{24}$ M. S. Child, Molecular Collision Theory (Academic, New York, 1974).

${ }^{25}$ M. Abramowitz and I. A. Stegun, Handbook of Mathematical Functions (National Bureau of Standards, Washington, D.C., 1964).

${ }^{26}$ A. van der Avoird, P. E. S. Wormer, and R. Moszynski, Chem. Rev. 94, 1931 (1994).

${ }^{27}$ W. Klopper, M. Quack, and M. A. Suhm, J. Chem. Phys. 108, 10096 (1998)

${ }^{28}$ B. R. Johnson, J. Chem. Phys. 67, 4086 (1977).

${ }^{29}$ F. X. Gadéa, H. Berriche, O. Roncero, P. Villarreal, and G. D. Barrio, J. Chem. Phys. 107, 10515 (1997).

${ }^{30}$ R. Sileo and T. Cool, J. Chem. Phys. 65, 117 (1976).

${ }^{31}$ M. Quack and M. A. Suhm, J. Chem. Phys. 95, 28 (1991). 